\title{
PHOTOCHROMIC ORGANIC-INORGANIC HYBRID NANOCOMPOSITE HARD COATINGS WITH TAILORED FAST SWITCHING PROPERTIES
}

\author{
M. Mennig, K. Fries and $H$. Schmidt \\ Institute for New Materials, GmbH, Im Stadtwald 43, D- 66123 Saarbruecken, Germany
}

\section{ABSTRACT}

A recently developed photochromic organic-inorganic nanocomposite (Nanomer ${ }^{\circledR}$ ) coating system is investigated, which is based on an epoxysilane as network former, different bisepoxides as spacer and an organic amine as thermal cross-linker. This coating system is compatible with different photochromic dyes like oxazines, pyrans and fulgides and also with surface modified ceramic nanoparticles as filler in order to obtain macroscopic "hard properties" without changing the photochromic kinetics. In order to investigate the dye-spacer interactions and influence on the switching kinetic behavior, the matrix composition was diminished to the network former, the thermal cross-linker and two types (polar and nonpolar) of spacers in different concentrations. Four commercially available spirooxazines with different molecular sizes and polarities were incorporated into the matrices and coatings on glass slides were prepared by float-coating and cured at $130^{\circ} \mathrm{C}$ for $2 \mathrm{~h}$. The half darkening and half fading times of the coatings were measured by a fast diode array spectrometer as a function of spacer type and concentration for the four different dyes. The "nonpolar" spacer CHMG did not influence the switching kinetic of the dyes significantly and fast switching times of $2 \mathrm{~s}-4 \mathrm{~s}$ were obtained. The "polar" spacer PCF (aromatic character) showed interactions with the dye molecules, which led to an increase in the switching times of up to $25 \mathrm{~s}$. The retardation of the switching process increased with increasing spacer content and with increasing polar character of the dye molecule. From these results, it could be concluded that the photochromic behavior of the spirooxazines could be adjusted to different applications by interactions with polar spacers.

\section{INTRODUCTION}

Photochromism is the reversible color change undergone by a compound under UV irradiation. The photochromic phenomenon is exhibited by two different classes of materials. Metal halides in melted special glasses are used as active materials for ophthalmic applications, but offer only a limited color range and are not suitable for treating organic polymeric substrates. In addition, organic dyes like spirooxazines, spiropyrans, chromenes and dihydroindolizines in organic polymers show a photochromic effect [1], and photochromic polymeric lenses have been obtained with organic photochromic dyes via a thermal transfer technique $[2,3]$. Disadvantages are high processing costs, switching times $>100 \mathrm{~s}$ and the need for a hard topcoat.

The ring opened, colored form of spirooxazines and spiropyrans may interact with their environment, i.e. matrix, leading to different photochromic responses $[4,5]$. The important role of the dye-matrix interactions was first investigated by Levy and co-workers $[6,7]$ in the photochromic behavior of spiropyrans: the open zwitterionic colored forms of the dyes are probably stabilized in hydrophilic matrix domains (containing residual $\mathrm{S}-\mathrm{OH}$ groups), which could lead to reverse photochromism. In contrast, the closed, colorless form is more stable in hydrophobic sol-gel matrix domains (polysiloxane chains with $\mathrm{CH}_{3}-$ or $\mathrm{C}_{2} \mathrm{H}_{5}$-groups) [8-12], which leads to direct photochromism. In organic-inorganic matrices containing hydrophilic and hydrophobic domains, a competition between direct and reverse photochromism can be 
observed [12]. In general, one may assume that a certain inner free volume of the host material would be required to avoid sterical hindering for the switching process. On the other hand, reactions of the photochromic dyes with moisture and oxygen from the environment, which lead to a decay of the photochromic activity [13] should be hindered by a minimum inner free volume of the host.

In order to fullfill these demands, the use of an organic-inorganic nanocomposite coating matrix with "hard" and "soft" microstructural units allows the development of photochromic coatings with sufficient free volume for fast switching dyes in combination with macroscopic hard properties (rigid structures). A model system for photochromic coatings on glass from 3glycidyloxypropyl-trimethoxysilane (GPTS), bisphenole A (BPA) and methylimidazole (MI) $[3,14]$ was used as a base line. Further developments in this field led to a new organicinorganic nanocomposite coating system, which is based on an epoxysilane as network former, different bisepoxides as spacers, and an organic amine as thermal cross-linker. This coating system is compatible with different photochromic dyes like oxazines, pyrans and fulgides and also with surface modified ceramic nanoparticles as filler in order to increase the scratch resistance without changing the photochromic kinetics [15]. Coatings with switching times of only $2 \mathrm{~s}-4 \mathrm{~s}$ were obtained [15], which are very short, compared to commercial photochromic eye glass lenses with switching times in the range of $1-3$ minutes. The fast switching behaviour can be very useful for applications in the fields of automotive or sports. However, for other application fields (ophthalmic or architecture) slower switching could be of interest.

Therefore, the general goal of this paper was to retard the photochromic behavior of spirooxazines by interactions with the nanocomposite system in order to adjust the kinetics to different applications without changing the direct photochromism into reverse photochromism [12]. To meet this goal, the polarity and amount of spacers was varied. As "probe" dyes, four spirooxazines with different polarity and molecular size were chosen. Ceramic nanoparticles, which do not influence the optical properties in the selected system [15], were not incorporated to keep the system simple at this stage. As an alternative, the decrease of the pore volume by different amounts of cross-linkers was investigated; these results will be published elsewhere [16].

\section{EXPERIMENTAL}

\section{Sol preparation}

For the preparation of the photochromic coatings, GPTS (3-glycidoxypropyltrimethoxysilane)-pre-hydrolysate [3] and TEOS (tetraethoxysilane) in molar ratio of 5:1; spacer (CHMG: cyclohexanedimethanoldiglycidyl, PCF: poly(phenylglycidylether)-coformaeldehyd) with molar ratio relative to GPTS $=0: 1,1 / 5: 1,2 / 5: 1,3 / 5: 1,4 / 5: 1$ and $1: 1$; $10 \mathrm{~mol} \%$ cross-linker IDA (isophorondiamine) relative to the epoxide groups; and $0.25 \mathrm{wt} \%$ photochromic dye (Blue $\mathrm{A}, \mathrm{Cl} 1, \mathrm{PC} 3, \mathrm{Cl} 2$ ) relative to the complete sol amount were mixed at room temperature. The maximum molar ratio between spacer and GPTS was 1:1, so that the role of GPTS as a network former in the hybrid material would not be suppressed too much with respect future to practical applications.

The investigated photochromic dyes were spirooxazines (Sigma-Aldrich):

Blue A: 1,3-dihydro-1,3,3-trimethyl-spiro[2H-indol-2,3'-[3H]-naphth-(2,1-b)(1,4)-oxazine], CL1: 5-chloro-1,3-dihydro-1,3,3-trimethyl-spiro[2H-indol-2,3'-[3H]-naphth-(2,1-b)(1,4)oxazine], PC3: 1,3-dihydro-1,3,3-trimethyl-spiro[2H-indol-2,3'-[3H]-phenanthr-(9,10-b)(1,4)-oxazine], CL2: 5chloro-1,3-dihydro-1,3,3-trimethyl-spiro[2H-indol-2,3'-[3H]-phenanthr- $(9,10-b)(1,4)$-oxazine]. 


\section{Preparation and characterization of the coatings}

Glass slides were cleaned in a dishwasher and float-coated with the photochromic sols and afterwards thermally cured at $130^{\circ} \mathrm{C}$ for $2 \mathrm{~h}$.

Characteristic photochromic properties, half darkening time $t_{E, 0.5}$ (time difference required for starting UV irradiation to increase to $50 \%$ of the maximum absorbance) and half-fading time $t_{\mathrm{A}, 0.5}$ (time difference required for the maximum absorbance to decay to $50 \%$ of the initial value after cut-off of the UV source) of these coatings were measured with a tempered diodearray UV-VIS spectrophotometer (HP 8453) using an external Xe-lamp (Osram XBO 450W/1) for irradiation ( $5 \mathrm{~mW} / \mathrm{cm}^{2}$ on sample surface) under isothermal conditions (room temperature). The samples were irradiated with UV-light for minimum $15 \mathrm{~s}$ (matrix without spacer, the fastest dye) up to about $200 \mathrm{~s}$ (matrix with maximum PCF content, the slowest dye) until the darkening was saturated. The ${ }^{13} \mathrm{C}-\mathrm{NMR}$ experiments were carried out on a Bruker AC 200 spectrometer. The positions of the NMR resonances were located taking $\mathrm{CDCl}_{3}$ as a reference. Chromacetylacetonate was used to reduce the relaxation time.

\section{RESULTS}

\section{Selection of the spacers}

A criterion was used to select spacers: organic molecule with at least two functional epoxide groups in order to react with the functional epoxide group of the network former by cross-linking.

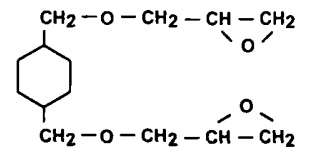

fig. 1: Molecular structure of cyclohexanedimethanoldiglycidyl (CHMG) [17]

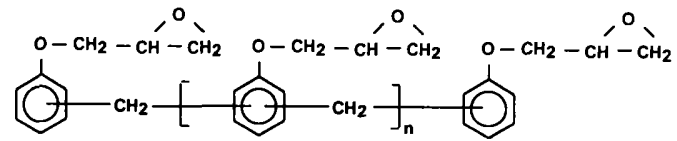

fig. 2: Molecular structure of poly(phenylglycidylether)-co-formaldehyde (PCF) with 2.2 epoxide groups per molecule [17]

The two figures above show the molecular structure of the selected spacers, which were commercially available. The bisepoxide CHMG (fig. 1) is the most nonpolar commercially available one (aliphatic, cyclic, 2 glycidyl-groups) and shows the most comparable molecular structure to the polar spacer PCF (fig. 2). Compared to CHMG, PCF obtains a polarity by the aromatic character (aromatic, cyclic, 2.2 glycidyl-groups).

It was proved by ${ }^{13} \mathrm{C}-\mathrm{NMR}$ spectroscopy that the epoxide groups of GPTS and spacer do not react under the chosen reaction conditions with water molecules (from the hydrolyzation of GPTS) within $2 \mathrm{~h}$ after adding these two sol components. For the coating preparation, the coating sols were applied onto the substrates within half an hour or less. Therefore it can be assumed that the starting conditions are similar for the investigation of the two different spacers in the matrix. 


\section{Darkening kinetics}

Figure 3 shows the darkening results.

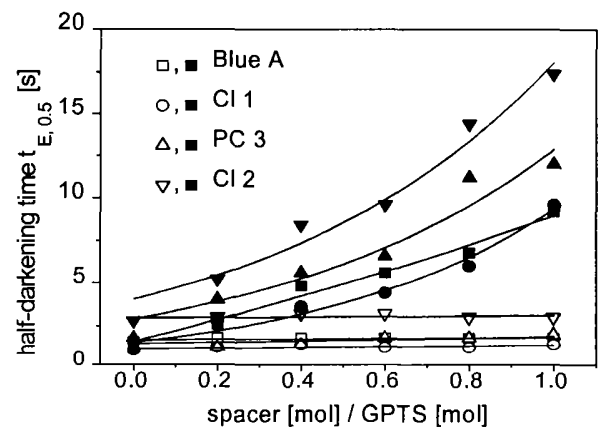

fig. 3: half-darkening time $\left(t_{E, 0.5}\right)$ of different spirooxazines in sol-gel matrices (with 10 mole\% cross-linker per epoxide group) on glass substrates (densified at $130^{\circ} \mathrm{C}$ for $2 \mathrm{~h}$ ) for different molar ratios of the spacer a) CHMG (unfilled markers, maximum error of measurement: $\pm 0.4 \mathrm{~s}$ ) and b) PCF (filled markers, Maximum error of measurement: $\pm 2.0 \mathrm{~s})$ to GPTS

Using CHMG in the matrix system with molar ratio to GPTS from 0:1 up to 1:1 (fig. 3a)), there was no significant influence of this nonpolar spacer on the half darkening times of the invested spirooxazines. Half darkening times $t_{E, 0.5}$ could be obtained between about $1 \mathrm{~s}(\mathrm{Cl} 1)$ up to about $3 \mathrm{~s}(\mathrm{Cl} 2)$. Using the polar spacer PCF in the same way (fig. $3 b)$ ), $t_{E, 0.5}$ of all photochromic dyes increased significantly up to $17 \mathrm{~s}(\mathrm{Cl} 2)$ with increasing amount of PCF (exponential fit in fig. 3b), kinetic first order [15]). In the frame of the errors of measurement for each graph in fig. 3 , there is no change in the half darkening times of the dyes in dependence on CHMG content. Consequently, the detected effects of PCF on the half darkening times cannot be attributed to the difference of the molecular size of the dyes in principle. The influence of PCF is much stronger on the half darkening times of $\mathrm{PC} 3$ and $\mathrm{Cl} 2$ than on those of Blue $\mathrm{A}$ and $\mathrm{Cl}$ 1. This can be explained in terms of the molecular structure of the different spirooxazines (fig. 4) all of which are indolino-spirooxazines.<smiles>[X]c1ccc2c(c1)C(C)(C)C1(C=Nc3c(c4ccccc4c4ccccc34)O1)N2C</smiles>

\begin{tabular}{l|c|c|c} 
& $X$ & $Y$ & $\begin{array}{c}\text { colour under } \\
\text { UV light }\end{array}$ \\
\hline Blue A & H & - & turquois \\
\hline Cl 1 & $\mathrm{Cl}$ & - & pale blue \\
\hline PC 3 & $\mathrm{H}$ & $\mathrm{C}_{4} \mathrm{H}_{4}$ & dark blue \\
\hline Cl 2 & $\mathrm{Cl}$ & $\mathrm{C}_{4} \mathrm{H}_{4}$ & blue-violet
\end{tabular}

fig. 4: structural formula of the photochromic dyes (spirooxazines) [17]

Both Blue $\mathrm{A}$ and $\mathrm{Cl} 1$ contain a naphtho-condensed oxazine ring and differ only in the $\mathrm{Cl}-$ substituted indolino-ring. In contrast to this, $\mathrm{PC} 3$ and $\mathrm{Cl} 2$ show a phenanthro-condensed oxazine ring, but also differ in the substitution of a $\mathrm{Cl}$ atom. Consequently, Blue $\mathrm{A}$ is the 
smallest and the least polar molecule of these dyes. From $\mathrm{Cl} 1$ over $\mathrm{PC} 3$ to $\mathrm{Cl} 2$, the molecule size and the polar character increase. It may be assumed that the dye-spacer interactions increase with increasing polarity of the dye molecule and/or of the spacer. By these dyematrix(spacer) polar interactions, the UV light-induced opening of the oxazine ringcycle (i.e., the coloring of the dye) is hindered and time retarded.

\section{Fading kinetics}

The coatings were darkened until saturation (within $15 \mathrm{~s}$ without spacer until $200 \mathrm{~s}$ with maximum PCF content) and the fading process was detected spectroscopically. The results are presented in fig. 5 .

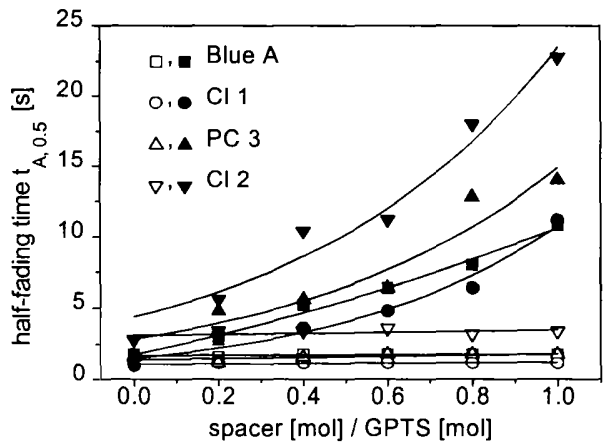

fig. 5: half-fading time $\left(t_{A, 0.5}\right)$ of different spirooxazines in sol-gel matrices (with 10 mole\% cross-linker per epoxide group) on glass substrates (densified at $130^{\circ} \mathrm{C}$ for $2 \mathrm{~h}$ ) for different molar ratios of the spacer a) CHMG (unfilled markers, maximum error of measurement: $\pm 0.4 \mathrm{~s}$ ) and b) PCF (filled markers, Maximum error of measurement: $\pm 2.0 \mathrm{~s}$ ) to GPTS

For the investigated spirooxazines, half fading times $t_{A, 0.5}$ between $1 \mathrm{~s}(\mathrm{Cl} 1)$ up to $3 \mathrm{~s}$ (Cl 2) could be obtained in the matrix system without a spacer (fig. 5). By the incorporation of CHMG into the matrix network (fig. 5a)), there is no effect on the half fading times of the dyes with increasing amount of this "nonpolar" spacer up to 1:1 molar ratio to GPTS (same observation on half darkening times, see fig. 3a)). In contrast to that, increasing amounts of the polar spacer PCF (fig. 5b)) led to an increase of the half fading times up to $23 \mathrm{~s}$. It can be assumed that the reason for this effect is the polar interaction between dye and matrix as already discussed for the darkening process. But the influence on the half fading times is stronger than that on the half darkening times. This could be explained in terms of the ringopened molecular structure of the dyes in the darkened state. The opened colored form (merocyanine structure) contains an expanded $\pi$-system compared to the closed colorless form, which leads to more polarity and accordingly to stronger interactions with the spacer molecules. This may explain the further retardation of the fading.

\section{CONCLUSIONS}

The photochromic behaviour of dyes incorporated in the pores of a matrix system is dependent on the polarity of the pore. By the introduction of a polar spacer into the matrix network, the switching kinetics of the investigated spirooxazines could be time retarded by dye-spacer interactions, i.e. the half fading times could be adjusted from $2 \mathrm{~s}$ up to $23 \mathrm{~s}$. In 
contrast to the study of Levy and co-workers $[6,7]$ and Sanchez and co-worker $[4,5]$, the photochromism of the dyes was not turned to reverse photochromism.

In future experiments the influence of the dye-spacer interactions on the UV long term stability of the dyes will be investigated. It can be expected that the polar interactions between the aromatic rings of the dye and spacer molecules look like a "sandwich structure", so that the dye molecule is protected by the spacer and radical or ion attacs, that would lead to dye degradation, are sterically hindered.

\section{ACKNOWLEDGEMENT}

The authors wish to thank the Federal Ministry of Research and the State of Saarland for financial support.

\section{REFERENCES}

[1] Photochromism, Molecules and Systems, H. Dürr, H. Bouas-Laurent, eds., Elsevier, Amsterdam, Oxford, New York, Tokyo (1990)

[2] J.-P. Crano, S. Weber, P.Tardieu, N. Lelard, WO 96/27488 (1996)

[3] L. Hou, B. Hoffmann, M. Mennig, H. Schmidt, presented at the 17 th International Congress on Glass, Oct 9-14 (1995), Beijing, China

[4] B. Schaudel, C. Guermeur, C. Sanchez, K. Nakatani and J. A. Delaire, J. Mater. Chem. 7 (1), 61 (1997)

[5] C. Sanchez, Proceeding of The Sixth International Symposium on New Glass, Oct. 14. 15., 1997, Kikai - Shinko - Kaikan, p 13-35

[6] D. Levy and D. Avnir, J. Phys. Chem. 92, 734 (1988)

[7] D. Levy, S. Einhorn and D. Avnir, J. Non-Cryst. Solids 113, 137 (1989)

[8] D. Preton, J. C. Pouxviel, T. Novinson, W. C. Kaska, B. Dunn and J. I. Zink, J. Phys. Chem. 94, 4167 (1990)

[9] H. Nakazumi, R. Nagashiro, S. Matsumoto and K. Isagawa, SPIE Proc. Vol. 2288, SolGel Optics III, San Diego, 1994

[10] L. Hou, B. Hoffmann, M. Mennig and H. Schmidt, J. Sol-Gel Sci. Techn. 2, 635 (1994)

[11] L. Hou, M. Mennig and H. Schmidt, Proc. Eurogel '92, 173 (1992)

[12] J. Biteau, F. Chaput and J. P. Boilot, J. Phys. Chem. 100, 9024 (1996)

[13] V. Malatesta, Book Chapter 10 for "Photochromic and Thermochromic Compounds", Plenum Press, No.4, USA (1997)

[14] L. Hou, B.- Hoffmann, H. Schmidt, M. Mennig, Sol-Gel Sci. Techn. 8, 923 (1997) and 927 (1997)

[15] M. Mennig, K. Fries and H. Schmidt, Proc. $2^{\text {nd }}$ Int. Conf. Coatings on Glass ICCG, Saarbrücken 1998, accepted for publication in Thin Solid Films, 1999

[16] M. Mennig, K. Fries and H. Schmidt, Deutsche Glastechnische Berichte, 1999 (accepted for publication)

[17] catalogue information of Sigma-Aldrich 